\title{
Clinical management of suspected intracranial meningioma in three dogs with pulsed high-dose hydroxyurea
}

\author{
Jae-Geum Cho, Kun-Ho Song, Kyoung-Won Seo* \\ Department of Veterinary Internal Medicine, College of Veterinary Medicine, Chungnam National University, \\ Daejeon 34134, Korea
}

(Received: March 21, 2018; Accepted: April 5, 2018)

\begin{abstract}
Three aged dogs, all over 15 years old, with multiple seizure events and various neurologic signs such as head turn and ataxia were brought to the referring hospital. Two cases were tentatively diagnosed with intracranial meningioma based on magnetic resonance imaging, and one case was histopathologically confirmed as meningioma at necropsy. All dogs improved after initiation of pulsed high-dose hydroxyurea (HU) treatment. Clinical sign relapses were managed by repeating high-dose HU without obvious side effects. Pulsed high-dose HU can be applied when clinical signs are refractory to the traditionally recommended dosage for intracranial meningioma in dogs.
\end{abstract}

Keywords: brain neoplasms, chemotherapy, hydroxyurea, meningioma, seizures

Meningioma is an extra-axial, primary tumor that originates from the meninges surrounding the brain, and comprises a large portion of space-occupying lesions in the cranium, with a prevalence of approximately $33-49 \%$ of primary brain tumors in aged dogs (median age, 9 years) $[1,3$, 5]. Meningioma is generally considered as a benign tumor; however, $33 \%$ of cases in dogs is accompanied by invasion, unlike in other species [9]. Therefore, dogs with meningioma should be thoroughly evaluated using a tumor grading system to determine the accurate prognosis $[4,6,7,10]$. The World Health Organization (WHO) international histological classification system was modified for use in dogs and includes the following grades: Grade 1 (benign), Grade 2 (atypical), and Grade 3 (malignant) [2, 4, 9].

The most common clinical signs of intracranial meningioma in dogs include seizures, ataxia, behavioral changes, visual deficits, circling, and other various neurologic dysfunctions $[1,4,6]$. Neurologic signs vary depending on the location and size of the tumor, and secondary effects such as inflammation, edema, and intracranial hypertension can also add to the severity of signs in the patient $[3,9]$.

Treatment options for intracranial meningioma in dogs can be divided into three types as follows: definitive (surgery and radiation), palliative (chemotherapy, anticonvulsant therapy, and decompressive agents), and alternative (gene, immune, and hormonal therapies) [1, 5, 9]. Surgical removal with adjunctive radiotherapy is the treatment of choice for improving patient quality of life and providing longer survival times
(16-30 months) [1, 2, 4, 6, 9]. However, most cases are limited to only palliative treatment due to cost, owner compliance, high risk of anesthesia, and surgical inaccessibility of the tumor. As chemotherapeutics penetrating the blood brain barrier, hydroxyurea (HU) is reportedly used for meningioma as an adjunctive or sole therapy, since it is inexpensive and has minimal and reversible side effects in veterinary medicine $[1,4]$. The traditional dosage of HU reported for the treatment of intracranial meningioma in dogs is 20-30 $\mathrm{mg} / \mathrm{kg}$ daily, with or without glucocorticoids for vasogenic edema $[4,7,8]$. However, a number of studies in veterinary medicine have shown that managing clinical signs with the traditional dosage is more difficult as time progresses [3, 9].

A 19-year-old spayed female Maltese (Case 1) with a history of several generalized tonic-clonic seizures was presented neurologically stable to the Veterinary Teaching Hospital of Chungnam National University. The owner complained that the patient had displayed intermittent seizures and neurologic signs, such as altered mentation and circling, for the past year. On magnetic resonance imaging (MRI), a homogenous contrast mass $(2.5 \times 1.4 \mathrm{~cm})$ with contrast enhancement in the right frontal lobe and olfactory bulb, along with an additional mass in the right nasal cavity, was identified and was accompanied by edema and compression of the lateral ventricle (Fig. 1A and B), and the patient was diagnosed with a probable intracranial meningioma, based on MRI features. Concurrent degenerative conditions, mitral valve diseases (ACVIM stage B2: hemodynamically significant valve

*Corresponding author

Tel: +82-42-821-6756, Fax: +82-42-821-6703

E-mail: kwseo@cnu.ac.kr 


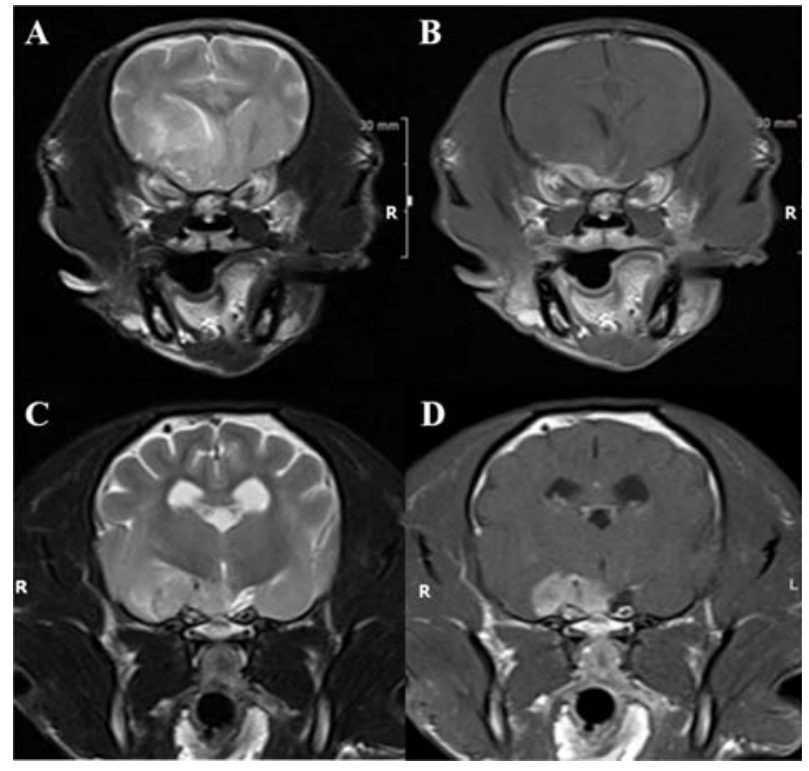

Fig. 1. A 19-year-old female Maltese (Case 1) with several generalized tonic-clonic seizures. Homogenous contrast mass $(2.5 \times$ $1.4 \mathrm{~cm}$ ) with contrast enhancement in right frontal lobe and edematous lesion and compression of lateral ventricle in T2weighted transverse image (A) and T1-weighted transverse image (B). A 16-year-old castrated male Yorkshire Terrier (Case $3)$ after the second cluster seizure episodes. Mass $(1.2 \times 1.0 \mathrm{~cm})$ formed from meninges at the level of optic chiasm and hypothalamus with peritumoral edema in $\mathrm{T} 2$-weighted transverse image (C) and T1-weighted transverse image, post contrast (D).

regurgitation but asymptomatic) and chronic kidney disease (IRIS stage 2: creatinine $1.4-1.8 \mathrm{mg} / \mathrm{dL}$ ), were also diagnosed. Palliative chemotherapy was chosen in this case, since surgery was declined due to the difficulty of accessing the lesion. The patient's neurologic status gradually improved with a high dose of $\mathrm{HU}(50 \mathrm{mg} / \mathrm{kg} /$ day $)$, dexamethasone $(0.25 \mathrm{mg} / \mathrm{kg} / \mathrm{day})$, and an anticonvulsant during the initial 7 days and stabilized while the dosage was reduced to $20 \mathrm{mg}$ / $\mathrm{kg} /$ day. However, the patient died of cardio-renal syndrome with respiratory failure 4 months later.

A 16-year-old intact female Shih Tzu (Case 2) in status epilepticus and with a right-sided head turn was referred and the clinical signs had been worsening over the previous 2 years according to the owner. Along with multiple seizure episodes, a variety of neurologic signs, such as altered mentation, head tilt, and nystagmus, were observed in this case. On MRI, a mass with a dural tail sign $(2.1 \times 3.0 \mathrm{~cm})$ in the right temporal lobe was identified and was compressing the brainstem and cerebellum, inducing severe peritumoral edema in the frontal lobe and mesencephalon (Fig. 2A). After stabilizing the status epilepticus, high-dose HU (50 mg/ $\mathrm{kg} /$ day for 5 days) was administered, along with dexamethasone $(0.25 \mathrm{mg} / \mathrm{kg} / \mathrm{day})$, and the patient's neurologic status and general condition dramatically improved to a near-normal state. Furthermore, a decrease in size of the mass $(1.7 \times$

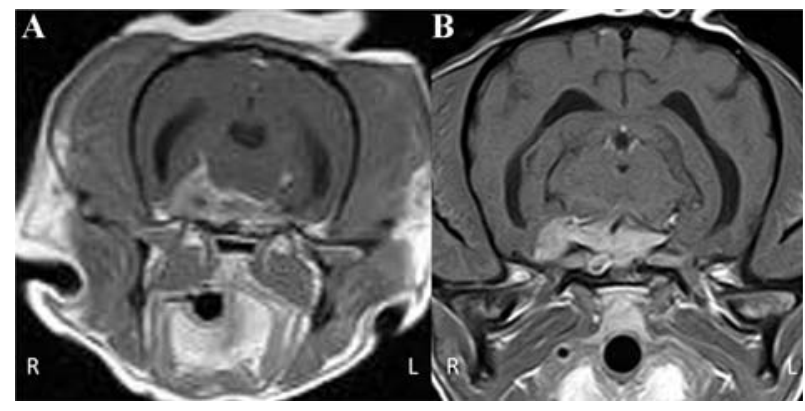

Fig. 2. A 16-year-old intact female Shit Tzu (Case 2) with presentation of status epilepticus and right head turn. Mass with dural tail sign $(2.1 \times 3.0 \mathrm{~cm})$ on right temporal lobe in T1weighted transverse image $(\mathrm{A})$ and decreased mass size $(2.1 \times$ 3.0 to $1.7 \times 1.2 \mathrm{~cm}$ ) at 6 months after $\mathrm{HU}$ and dexamethasone combination therapy T1-weighted transverse image, post-contrast (B).

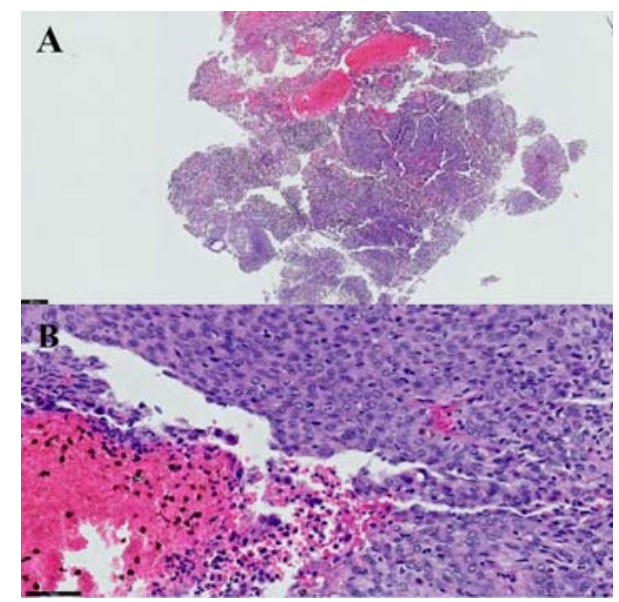

Fig. 3. A 16-year-old intact female Shit Tzu (Case 2). Brain tissues obtained at necropsy representing inflamed and partially necrotic meningioma. H\&E stain. $50 \times($ A) and $400 \times(B)$.

$1.2 \mathrm{~cm}$ ) and peritumoral edema was noted on MRI recheck 6 months into treatment (Fig. 2B). When seizures or other neurologic signs occasionally recurred, the high-dose HU was again administered and then tapered back to the traditional dose $(20-30 \mathrm{mg} / \mathrm{kg} /$ day $)$, and the dog managed well with the adjustments. She survived for 10 months and was then euthanized due to uncontrollable neurological dysfunctions, and the diagnosis of an inflamed and necrotic intracranial meningioma was confirmed by histopathological evaluation of biopsy samples taken at necropsy (Fig. 3).

A 16-year-old castrated male Yorkshire Terrier (Case 3) was presented after a second episode of cluster seizures, and the owner reported an abrupt onset of signs. On initial screening, hepatic neoplasia (suspected biliary adenoma) was identified, and MRI of the brain revealed a mass $(1.2 \times 1.0 \mathrm{~cm})$ originating from the meninges at the level of the optic chiasm and hypothalamus with moderate peritumoral edema (Fig. 1C and D). The owner declined surgical treatment due 
to anesthetic risk, so immediate high-dose HU treatment (50 $\mathrm{mg} / \mathrm{kg} /$ day for 5 days) was initiated, and the dose was tapered to the maintenance dose $(25 \mathrm{mg} / \mathrm{kg} /$ day to every other day). This patient is still alive (15 months since the first treatment) and is neurologically normal receiving intermittent high-dose HU and an anticonvulsant drug. This study received the approval from Chungnam National University Institutional Animal Care and Use Committee (CNUA-18-0084) and obtained owners' consents.

In the present study, three dogs older than 15 years at diagnosis were suspected of having intracranial meningioma based on MRI findings along with various clinical signs, and all patients showed secondary effects, such as peritumoral edema and compression of the parenchyma on MRI. Notably, the dog that was in status epilepticus at presentation (Case 2) had more severe edema than the other cases on MRI.

The goal of therapy for dogs with intracranial meningioma is alleviation of neurologic signs [4, 6]. Although HU is recommended as an adjunctive treatment in the literature, it has been used in many intracranial meningioma cases in veterinary practices without definitive therapy $[6,9]$. In one study, neurologic signs of a patient were alleviated with a standard dose $(30 \mathrm{mg} / \mathrm{kg}$ every other day) of HU and dexamethasone $(0.5 \mathrm{mg} / \mathrm{kg} /$ day $)$, and the patient's status was stable enough to pursue follow-up MRI after a month. The results demonstrated a decrease in tumor size and peritumoral edema. However, clinical signs recurred 7 months into treatment, and MRI showed that the tumor had regrown to its original size without edema. The patient was euthanized due to the severe neurologic status [11]. Though many studies have shown the initial effectiveness of HU, clinical signs often recur after certain time periods, suggesting that the tumor becomes refractory to the standard dosage $[5,9]$.

Pulse therapy, which involves intermittent administration of high-dose medication, is often used with chemotherapies for maximizing the therapeutic effect and minimizing the side effects. In the cases presented in this study, intermittent high-dose HU was administered based on the neurological signs in each case. HU at a dose of $50 \mathrm{mg} / \mathrm{kg}$ once a day was initiated, along with dexamethasone $(0.25 \mathrm{mg} / \mathrm{kg} /$ day $)$ in all cases, and the HU was then tapered to 25 to $30 \mathrm{mg} / \mathrm{kg}$ once a day with the improvement of clinical signs. All three patients were stable while being treated with pulse therapy of highdose HU and were well managed for 4, 10, and 15 months, respectively. In Case 2, though the patient intermittently displayed mild neurological deficits, such as dullness and head tilt in the course of treatment, the dog's general condition was stable, and he survived for 10 months. Furthermore, only reversible adverse effects of $\mathrm{HU}$, such as mild anemia, alopecia, and pigmentation, were identified. In Case 1, the patient was managed without any serious neurologic dysfunction until she died of pulmonary edema caused by congestive heart failure. Since the patient was 19 years old, her general condition progressively deteriorated due to concurrent dis- eases. The patient in Case 3 has survived for 15 months to date, with intermittent seizures that typically occur when the owner neglects to give a dose of the medication. The patient's quality of life remains good with the current treatment combination of $\mathrm{HU}(30 \mathrm{mg} / \mathrm{kg} /$ day $)$ and an anticonvulsant drug except skin pigmentation noted at 7 months.

In our study the administered high-dose of HU was $50 \mathrm{mg} /$ $\mathrm{kg}$ /day for 5 to 7 days. However, it was adjusted depending on the clinical signs of each patient and tapered to the standard dose $(20-30 \mathrm{mg} / \mathrm{kg} /$ day to every other day) for maintenance. Severe side effects enough to cause discontinuation of the pulse therapy were not observed during the entire treatment period.

In conclusion, based on the survival times and patients' quality of life in our study, pulse high-dose therapy of HU could be considered as a reasonable alternative therapeutic option, along with other treatments, such as proper decompression, anticonvulsants, and anti-inflammatory drugs [6-8]. Pulse therapy may be especially useful in relapsed cases or those refractory to the standard dosage of HU, and it could help to improve not only patient status but also survival time.

The limitations of this study include that only one of the cases was histopathologically confirmed as intracranial meningioma, the number of cases was small, and the response to treatment and survival time of meningioma vary depending on the location and size of the tumor. Therefore, further study is needed to investigate the effects of pulse high-dose therapy of HU in a larger number of canine intracranial meningioma cases.

\section{References}

1. Adamo PF, Forrest L, Dubielzig R. Canine and feline meningiomas: diagnosis, treatment, and prognosis. Compend Contin Educ Vet 2004, 26, 951-966.

2. Dickinson PJ. Advances in diagnostic and treatment modalities for intracranial tumors. J Vet Intern Med 2014, 28, 11651185.

3. Ettinger SJ, Feldman EC. Neurologic disease. In: Textbook of Veterinary Internal Medicine. 8th ed. pp. 1363-1464, Saunders, St. Louis, 2017.

4. Horta RS, Martins BC, Martins GC, Marcinowska A. Advances in diagnosis and management of intracranial and spinal meningiomas in dogs and cats. Rev Port Cienc Vet 2015, 110, 172-180.

5. LeCouteur RA, Withrow SJ. Tumors of the nervous system. In: Withrow and MacEwen's Small Animal Clinical Oncology. 4th ed. pp. 583-589, Elsevier Saunders, St. Louis, 2007

6. Motta L, Mandara MT, Skerritt GC. Canine and feline intracranial meningiomas: an updated review. Vet J 2012, 192, 153-165.

7. Newton HB. Hydroxyurea chemotherapy in the treatment of meningiomas. Neurosurg Focus 2007, 23, E11.

8. Schrell UMH, Rittig MG Anders M, Koch UH, Marschalek R, Kiesewetter F, Fahlbusch R. Hydroxyurea for treatment of unresectable and recurrent meningiomas. II. 
Decrease in the size of meningiomas in patients treated with hydroxyurea. J Neurosurg 1997, 86, 840-844.

9. Sessums K, Mariani C. Intracranial meningioma in dogs and cats: a comparative review. Compend Contin Educ Vet 2009, 31, 330-339.

10. Sturges BK, Dickinson PJ, Bollen AW, Koblik PD, Kass PH, Kortz GD, Vernau KM, Knipe MF, LeCouteur RA,
Higgins RJ. Magnetic resonance imaging and histological classification of intracranial meningiomas in 112 dogs. J Vet Intern Med 2008, 22, 586-595.

11. Tamura S, Tamura Y, Ohoka A, Hasegawa T, Uchida K. A canine case of skull base meningioma treated with hydroxyurea. J Vet Med Sci 2007, 69, 1313-1315. 